\title{
The interconnection exchange and complex systems properties in power grid network
}

\author{
Piotr Hadaj ${ }^{*}$, Marek Nowak ${ }^{1}$, and Dominik Strzałka ${ }^{1}$ \\ ${ }^{1}$ Faculty of Electrical and Computer Engineering, Rzeszów University of Technology, Al. \\ Powstańców Warszawy 12, 35-959 Rzeszów, Poland
}

\begin{abstract}
A case study based on the real data obtained from the Polish PSE System Operator of the highest voltages electrical energy network is shown. The data about the interconnection exchange and some complex networks (graphs) parameters were examined, after the removal of selected nodes. This allowed to test selected network parameters and to show that the breakdown of only three nodes in this network can cause significant drop of its average efficiency.
\end{abstract}

\section{Introduction}

The concept of complex systems and related theory give many interesting applications in modelling different real systems [1]. One of examples are power systems which can be exposed on different threats even leading to the risk of potential blackouts [2]. In this short paper, the real electrical network as a graph of nodes and edges is shown. Some of this network topological parameters are given and the interconnection exchange as an example of the power system security is shown. Selected parameters of complex networks are calculated and the removal of some network nodes caused by failure was done. This leads to the changes in the whole network topology shown in tables. The most important is the drop of network efficiency and in turn, the increase of transmission costs.

The paper is organized as follows: after the short Introduction in Section 2 we show the complex systems and networks theory. Section 3 gives the shape of the EU-PL electricity exchange system. The analysis of real source data based on Network Workbench and Gephi software packages is given in Section 4 . Section 5 shows the paper summary.

\section{Complex systems and networks}

The idea of complex systems refers to the concept of systems $S$ understood as beings $B$, which consist of $n$ elements $E=\left\{e_{1}, e_{2}, \ldots, e_{n}\right\}$, that pose $m$ attributes $A=\left\{a_{1}, a_{2}, \ldots, a_{m}\right\}$ with $k$ possible long- or short-range relations $R=\left\{r_{1}, r_{2}, \ldots, r_{k}\right\}$, finally giving $S=B(E, A$, $R$ ) $[3,4]$. Usually, the definition of complex systems is referred to the Aristotle's rule: the whole is more than the sum of its parts [5].

* Corresponding author: piotr.hadaj@prz.edu.pl 
Complex systems show many interesting features in both: spatial and temporal domains. Among them there are complex networks of nodes and relations (edges) based on graphs $G$ $=\{V, E\}$ with $n$ nodes (vertices $\left.V=\left\{v_{1}, v_{2}, \ldots, v_{n}\right\}\right)$ connected by $m$ edges $E=\left\{e_{1}, e_{2}, \ldots\right.$, $\left.e_{m}\right\}$. Such graphs are usually characterized by special features. The length of the shortest path $d_{i j}$ calculated as the sum of the $\ell_{i j}$ distances (paths) between all possible paths in the graph between the nodes $i$ and $j, d_{i j} \geq \ell_{i j}, \forall i \neq j$ is usually quite low even then the size of network (its diameter) is big. It can be used to measure the scattering of the network [6]. The parameter defined as $\varepsilon_{i j}=1 / d_{i j}, \forall i \neq j$ can represent the network efficiency (if $d_{i j}=\infty$, then $\varepsilon_{i j}=0$ ). The high network efficiency is high means low costs of its operation (e.g. costs of energy transmission during the interconnection exchange). If network of vertices has $k_{i}$ edges that connect each vertex to other $k_{i}$ nodes then the ratio between the number $E_{i}$ of edges that exist among these $k_{i}$ nodes and the number of total possible vertices connections, give the clustering coefficient $C_{i}$ of node $i$. For clique $C_{i}=1$, for random graphs $C_{i} \rightarrow 0$, but for complex networks this parameter can vary [7]. Graphs with high value of $C$ are called small worlds.

\section{The interconnection exchange between systems}

The interconnection exchange is an inseparable element of the power system operation. From the system point of view, it allows, during the high demand, to import energy from another county in order to provide electricity supply to consumers, and to transfer energy to another country when the surplus energy is produced. The existence of such connections significantly increases the energy security of the power system. This is the key future of plans related to the energy independence and energetic security in different countries of the EU (European Union). However, in order to assume that the whole system will be stable one of the key aspects is the quality of the supplied energy. The fluctuations in RMS (root mean square) of voltage value, high harmonic content, or frequency fluctuations [8] should be as low as possible. Moreover, the supply reliability and continuity shown by SAIDI parameter (System Average Interruption Duration Index) or SAIFI (System Average Interruption Frequency Index) parameter are of great importance [8]. If the topology of power networks nodes connections is resistant to the net damages caused by different random events then the country economy is attractive for investors $[9,10]$. In Poland the PSE as the National System Operator, is responsible for the reliability of the highest voltages energy networks, while the local Distribution System Operators (DSO) are responsible for the remaining high-, medium-, and low-voltage lines.

Currently, the HVDC bridges are used as interconnections, such as the connection of Poland and Sweden via the Swe-Pol Link sea line, and the HVDC Back-to-Back solution in the connection of the Polish and Lithuanian systems (LitPol Link). These are expensive and often difficult to implement, but they replace traditional solutions (direct AC connections). It turns out, however, that under certain conditions such a transmission is much more advantageous and provides an acceptable return on investment. The HVDC SwePol Link HVDC line, launched in year 2000, provides a combination of Polish and Swedish systems. It is implemented as an undersea cable line that connects two converter stations connected to the Polish and Swedish systems. The connection between the Polish and Lithuanian systems is not a typical HVDC bridge. This connection consists of only one converter station, which connects the systems. This connection has most of the advantages of a typical HVDC bridge and is used when the systems between which the energy exchange is to take place are not synchronized (as in this case). Figure 1 shows the annual energy exchange balance from the European Network of Transmission System Operators for Electricity report and the graph of all energy exchanges in Europe [11]. 

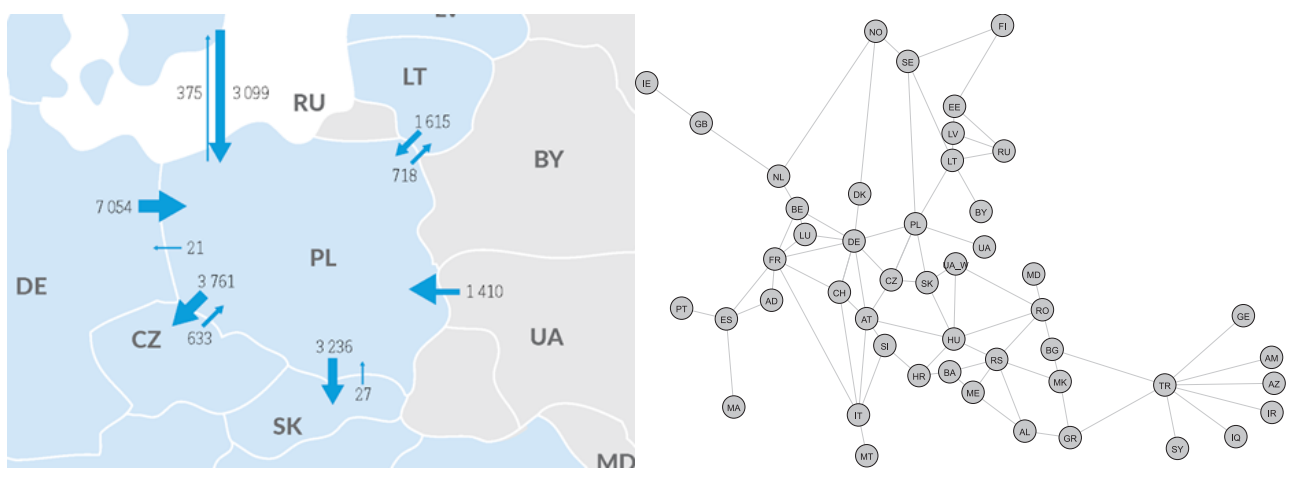

Fig. 1. Electricity exchange between Poland and neighboring countries in GWh (left) and power system interconnection in Europe as a graph of a complex network (right).

Table 1. Shows the energy exchange balance between Poland and neighbouring countries in GWh per year [11].

Table 1. Interconnection system exchange balance data for Poland and its neighbors.

\begin{tabular}{|c|c|c|c|c|c|c|c|}
\hline Year $A[G W h]$ & Germany & Czech Republic & Slovakia & Ukraine & Lithuania & Sweden & $\begin{array}{c}\text { Total } \\
\text { balance }\end{array}$ \\
\hline 2009 & -5483 & 6737 & 2274 & -199 & - & -1140 & 2189 \\
\hline 2010 & -5167 & 5364 & 1415 & 0 & - & -267 & 1345 \\
\hline 2011 & -4705 & 8208 & 3028 & -59 & - & -1236 & 5236 \\
\hline 2012 & -5877 & 8754 & 3498 & -1005 & - & -2545 & 2825 \\
\hline 2013 & -4909 & 7663 & 3051 & -1029 & - & -253 & 4523 \\
\hline 2014 & -9153 & 7158 & 3496 & -685 & - & -2984 & -2168 \\
\hline 2015 & -10644 & 9549 & 4926 & -67 & 50 & -3491 & 323 \\
\hline 2016 & -8740 & 6693 & 4185 & -957 & -596 & -2587 & -2002 \\
\hline 2017 & -7319 & 5571 & 4372 & -895 & -1042 & -2974 & -2287 \\
\hline 2018 & -7033 & 3128 & 3209 & -1410 & -897 & -2724 & -5727 \\
\hline 2019 & $\begin{array}{l}-7541 \\
\end{array}$ & $\begin{array}{l}-1810 \\
\end{array}$ & 1916 & -658 & $\begin{array}{l}-1401 \\
\end{array}$ & $\begin{array}{l}-1043 \\
\end{array}$ & -10537 \\
\hline
\end{tabular}

\section{Case study}

In this Section, the power grid based on PSE data is analyzed (see Fig. 2). All data has been entered into the Gephi software package and the real network consists of 124 vertices and 175 edges. Because of security reasons we are not able to show all details relating to node's physical location. But this graph is enough to examine the complex network features presented in Section 2. The nodes and edges in the graph refer to the physical objects in the transmission network: the nodes are transformers or power distribution stations, the edges are transmission lines.

Figure 3 shows the graphical analysis of the network (from Fig. 2) degree centrality. The count of the number of node connections is converted into a $0 . . .1$ scale (the node with the highest number has the value 1 whereas the other ones as the ratio of the largest one). The more blue is the node's color, the more important is the node.

Now we are going to remove some nodes from the network starting from the nodes with the highest degrees. We assume that when it is done this could cause the major negative effects (for example after the terroristic attack). After each removal the graph properties are shown in Table 2. 


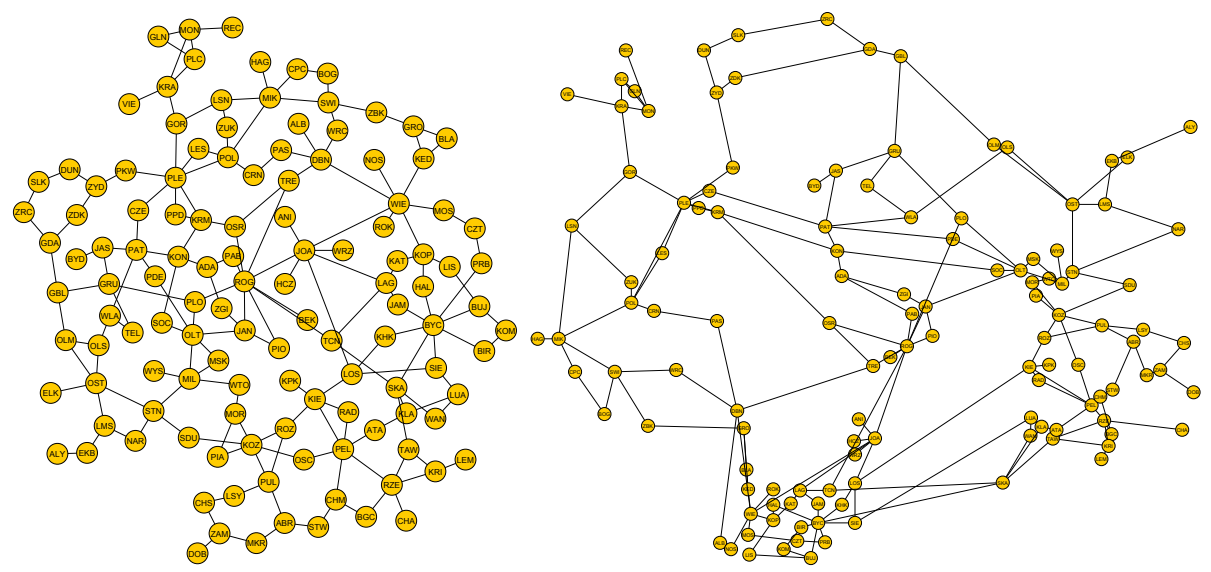

Fig. 2. Graph based on PSE power grid network: organic layout (left) and physical (geographical) layout (right).

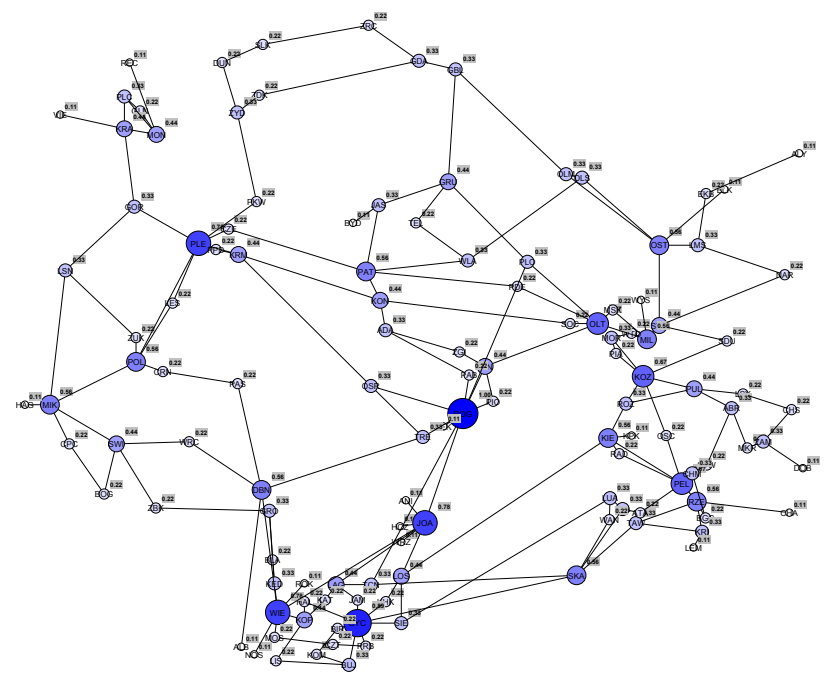

Fig. 3. The graph nodes centrality for Fig. 2 (right).

The description of parameters given in Table 2 can be found in literature, see for example [12]. It can be seen that after the removal of 3 nodes the average network efficiency falls more than $25 \%$.

Table 2. Averaged data from the analysis of the modified graphs.

\begin{tabular}{|c|c|c|c|c|}
\hline Number of Removed Nodes & 0 & 1 & 2 & 3 (avg.) \\
\hline Nodes in graph & 124 & 123 & 122 & 121 \\
\hline Edges in graph & 175 & 166 & 158 & 151 \\
\hline Average vertex degree & 2.823 & 2.699 & 2.59 & 2.5 \\
\hline Graph density & 0.023 & 0.022 & 0.021 & 0.021 \\
\hline Local efficiency & 0.114 & 0.1 & 0.101 & 0.097 \\
\hline Average efficiency & 0.216 & 0.156 & 0.191 & 0.171 \\
\hline Average clustering coefficient & 0.131 & 0.117 & 0.124 & 0.121 \\
\hline Average path length & 5.984 & 6.8 & 6.93 & 7.92 \\
\hline Graph diameter & 16 & 17 & 17 & 20.67 \\
\hline
\end{tabular}




\section{Summary}

This paper shows the data about the energy interconnection exchange and the analysis of complex network parameters of real data obtained from PSE network. The removal of some nodes in analyzed network caused the drop of network average efficiency by more than $25 \%$. The efficiency is related to the cost of whole network operation: high efficiency means low costs. In turn the breakdown, damage or accidents in the nodes of the highest degree can significantly increase the cost of whole network operation. We show that it is enough to break 3 vertices to cause such a situation.

Authors' alphabetical order means equal contribution.

\section{References}

1. Newman, M.; Networks: An Introduction; Oxford University Press, Inc.: New York, NY, USA, 2010.

2. Wu, L.; Anderson, R.N.; Boulanger, A.; Rudin, C.; Kaiser, G.E. Failure Analysis of the New York City Power Grid; CU CS Technical Report CUCS-025-14; Department of Computer Science, Columbia University: Columbia, 2014.

3. Klir, G.J. Trends in General Systems Theory; Wiley Interscience: New York, NY, USA, 1972

4. von Bertalanffy, L. General System Theory: Foundations, Development; George Braziller: New York, NY, USA, 1968.

5. Upton, J.; Janeka, I.; Ferraro, N. The whole is more than the sum of its parts: Aristotle, metaphysical. J Craniofacial Surg. 2014, 25, 59-63.

6. Å. J. Holmgren, Using Graph Models to Analyze the Vulnerability of Electric Power Networks, Risk Analysis, an International Journal 2006, 26, 955-969.

7. Latora, V.; Marchiori, M. Efficient behavior of small-world networks. Phys. Rev. Lett. 2001, 87, 198701.

8. Łatka, M.; Nowak, M. Analysis of Electrical Power Quality Parameters at the High Voltage Level. In Proceedings of the 2018 Conference on Progress in Applied Electrical Engineering (PAEE), Kościelisko, Poland, 18-22th June 2018.

9. Markovskii, P.; Merkushev, A. Investigation into high voltage network reliability predictive calculation. In Proceedings of the 2018 19th International Scientific Conference on Electric Power Engineering (EPE), Brno, Czech Republic, 16-18th May 2018.

10. Polish Ministry of Energy, Polityka Energetyczna Polski do 2040 Roku (PEP2040). ver. 1.2 2018. Available online: https://www.gov.pl/web/aktywapanstwowe/zaktualizowany-projekt-polityki-energetycznej-polski-do-2040-r (accessed on 9 May 2020).

11. European Network of Transmission System Operators for Electricity statistical reports: https://www.entsoe.eu/publications/statistics-and-data/\#statistical-factsheet (accessed on 15 May 2020).

12. Hadaj, P.; Strzałka D.: Modelling Selected Parameters of Power Grid Network in the South-Eastern Part of Poland: The Case Study, Energies, 2020,13 (1), 239 